\title{
Ganglion Cysts of the Proximal Tibiofibular Joint
}

\author{
Anthony Woodward, MD
}

The causes of foot drop or lumps around the knee can sometimes be challenging to diagnose in the primary care or internal medicine setting. This article aims to describe one cause of both conditions.

Ganglion cysts around the proximal tibiofibular joint at the knee may be asymptomatic, except for the presence of a mass, or cause neurologic problems by putting pressure on the adjacent peroneal nerve. The treatment and prognosis of cysts that cause neurologic deficit have been recently reviewed by Papanastassiou and colleagues.' Overall, 26 of 29 patients were men, and the median age of participants was 67 years. As expected, the cysts had low signal intensity on $\mathrm{T} 1$ sequences and high signal intensity on T2 sequences on magnetic resonance imaging (MRI) scans. Neurologic symptoms included peroneal palsy, pain, or paresthesia. Treatment was surgical excision of the cysts. In patients older than 65 years, peroneal palsy, if present, was treated by transfer of the posterior tibial tendon to the lateral cuneiform.

The authors followed 25 of the 29 patients for 1 to 10 years. Of the 25 patients, 4 had an intraneural ganglion; the remainder were extraneural. Eight of the patients had peroneal palsy. After the operation, 2 cysts had recurred but did not cause other symptoms. One patient had developed peroneal palsy following operative damage to the peroneal nerve. Two of the patients who had tendon transfers had developed soft tissue infections of the foot.

A PubMed search showed that other studies have reported a higher recurrence rate. Lateur and colleagues ${ }^{2}$ reported one recurrence after 4 primary excisions (25\%). Miskovsky and colleagues ${ }^{3}$ treated 8 patients with primary excision; one cyst (13\%) had recurred. In both studies, excision of recurrent cysts had failed: all cysts recurred again. Miskovsky and colleagues $^{3}$ fused the proximal tibiofibular joints in 4 patients ( 2 primary cases and 2 recurrent cysts) without any further recurrence.

Papanastassiou and colleagues ${ }^{1}$ attribute their low recurrence rate to preoperative planning based on the MRI-demonstrated

\section{AFFILIATIONS:}

Orthopedic Surgeon, Portland, Oregon

\section{CITATION:}

Woodward A. Ganglion cysts of the proximal tibiofibular joint. Consultant. Published online June 4, 2021. doi:10.25270/con.2021.06.00004

Received March 4, 2021. Accepted April 1, 2021.

\section{DISCLOSURES:}

The authors report no relevant financial relationships.

\section{CORRESPONDENCE:}

Anthony Woodward, MD, 11125 South West Lynnridge Avenue, Portland, OR 97225

(ahwoodward@hotmail.com)

extent of the cyst, meticulous dissection to the stalk of the cyst, and ligation of the stalk.

Ganglion cysts of the proximal tibiofibular joint may present with peroneal paralysis without a palpable mass. In such cases, electrodiagnostic studies can demonstrate the site of the lesion, and MRI can identify its cause.

\section{Conclusion}

Ganglion cysts of the proximal tibiofibular joint are rare. Helpful tests are MRI and electrodiagnostic studies. Possible treatments are aspiration, excision, ligation of the stalk, and/or arthrodesis. The study by Papanastassiou and colleagues ${ }^{1}$ suggests that meticulous dissection and the ligation of the stalk may be the best treatment for primary cysts. Excision alone of a recurrent cyst is rarely successful without arthrodesis of the proximal tibiofibular joint.

\section{References}

1. Papanastassiou ID, Tolis K, Savvidou O, Fandridis E, Papagelopoulos P, Spyridonos $\mathrm{S}$. Ganglion cysts of the proximal tibiofibular joint: low risk of recurrence after total cyst excision. Clin Orthop Relat Res. 2021;479(3):534-542. https://doi. org/10.1097/corr.0000000000001329

2. Lateur G, Pailhé $R$, Refaie $R$, et al. Ganglion cysts of the proximal tibiofibular articulation: the role of arthrodesis and combined partial fibula excision. Int Orthop. 2018;42(6):1233-1239. https://doi. org/10.1007/s00264-017-3542-y

3. Miskovsky S, Kaeding C, Weis L. Proximal tibiofibular joint ganglion cysts: excision, recurrence, and joint arthrodesis. Am J Sports Med. 2004;32(4):1022-1028. https:// doi.org/10.1177/0363546503258706 